\title{
Controlled growth and characterization of epitaxially-laterally-overgrown InGaN/GaN quantum heterostructures
}

\author{
Emre Sari ${ }^{1,4}$, Ozgun Akyuz', Eun-Geun Choi ${ }^{2}$, In-Hwan Lee ${ }^{2}$,Jong Hyeob Baek ${ }^{3}$, \\ and Hilmi Volkan Demir ${ }^{1,4, *}$ \\ ${ }^{I}$ Department of Electrical and Electronics Engineering, Department of Physics and UNAM -- National Nanotechnology Research Center. \\ Bilkent University, 06800 Bilkent, Ankara, Turkey; ${ }^{2}$ School of Advanced Materials Engineering, Research Center of Industrial Technology, \\ Chonbuk National University, Chonju 561-756, Korea, ${ }^{3}$ Korea Photonics Technology Institute, Gwangju,500-460 Korea ${ }^{5}$ School of \\ Electrical and Electronic Engineering, Microelectronics Division; School of Physical and Mathematical Sciences, Physics and Applied \\ Physics Division, Luminous! Semiconductor Lighting and Display Center of Excellence, Nanyang Technological University, Singapore, \\ 639798 Singapore \\ *Contact email: volkan@,stanfordalumni.org, hvdemir@,ntu.edu.sg
}

Crystal material quality is fundamentally important for optoelectronic devices including laser diodes and light emitting diodes. To this end epitaxial lateral overgrowth (ELO) has proven to be a powerful technique for reducing dislocation density in $\mathrm{GaN}$ and its alloys [1,2]. Implementation and design of ELO process is, however, critical for obtaining high-quality material with high-efficiency quantum structures for light emitters [3].

In this study, we present our systematic study on controlled lateral epitaxial overgrowth and characterization of InGaN/GaN quantum heterostructures using metal organic chemical vapor deposition (MOCVD). Here we correlate performance of these ELO-InGaN/GaN epi-structures using their room-temperature photoluminescence (RT-PL). Each of these epi-structures is grown on identical single-sided sapphire templates of 2 inch in diameter. The templates are comprised of $5 \mu \mathrm{m}$ thick $\mathrm{GaN}$ on c-plane sapphire substrate, each having a different epitaxial lateral overgrowth stripe pattern. The ELO patterns consist of plasma enhanced chemical vapor deposition (PECVD) grown SiN mask stripes of 4, 7 and $10 \mu \mathrm{m}$ in width [1-100] direction, separated by $4 \mu \mathrm{m}$ windows. During the ELO process, a low-dislocation-density, $7 \mu \mathrm{m}$ thick, n-type $\mathrm{GaN}\left(\mathrm{N}_{\mathrm{Si}} \sim 4 \times 10^{18} \mathrm{~cm}^{-3}\right)$ layer is grown on top of SiN stripes, together with targeted complete coalescence planes in the centers of the stripes. After the ELO process, we continue with the vertical growth of 5 pairs of $2.5 \mathrm{~nm}$ thick $\mathrm{InGaN}$ quantum wells and $7.5 \mathrm{~nm}$ thick $\mathrm{GaN}$ barriers, and $150 \mathrm{~nm}$ thick p-GaN $\left(\mathrm{N}_{\mathrm{Mg}} \sim 6 \times 10^{17} \mathrm{~cm}^{-3}\right)$ layer. In addition to the patterned templates of ELO process, we use an additional identical template with no pattern on it as the reference (negative control group). Epitaxial growths of all the samples are performed simulataneously using four of the six 2 inch sample holders.

We perform the photoluminescence characterization of our epi-structures using a He-Cd laser with an excitation wavelength of $325 \mathrm{~nm}$ at room temperature under identical experimental conditions. Moreover, in each of our measurements, we adjust the pump beam spot size and position such that we measure PL signal only coming from low dislocation density regions of our structures. We present these PL spectra of our quantum strucutres in Figures 1(a)-(d). We also show the analysis involving the PL peak intensities, PL peak wavelengths and full width at half maximum (FWHM) values in Figures 2 (a)-(c).We obtain PL peak wavelengths in the range from 460 to $473 \mathrm{~nm}$, which indicates that a close level of $\mathrm{InN}$ is incorporated into the quantum well structures of each sample. From the sample with $4 \mu \mathrm{m}$ mask size, we obtain PL emission with the highest peak intensity and the narrowest spectrum, which shows that fluctuation of InN concentration throughout the quantum wells is the lowest and the material quality of multiple quantum well structures is the best.

Moreover, we observe the longest peak wavelength for the $10 \mu \mathrm{m}$ mask sample. One of the possible reasons for that might be that this sample lacks complete coalescence, forming more of InN-rich regions compared to other samples. The reason for the fluctuations in the long wavelength tail of the reference sample might be similar, since during the lateral growth step of ELO the surface roughness of this sample increases, which in turn increases the number of InN-rich regions throughout the quantum well layer.

In conclusion, we presented our systematic study on controlled epitaxial growth and characterization of InGaN/GaN quantum structures with low defect densities, grown simultaneously using epitaxial lateral overgrowth technique with different mask and window widths. The epi-sample with $4 \mu \mathrm{m}$ mask and growth window widths yielded the hightest PL peak intensity with the narrowest spectrum.

This work is supported by NRF-RF, EU-FP7 Nanophotonics4Energy NoE, and TUBITAK EEEAG 107E088, 109E002, 109E004, and 110E010. HVD acknowledges support from ESF-EURYI and TUBA-GEBIP, and ES, from TUBITAK-BIDEB.

\section{References}

[1] A. Usui et al., Jpn. Jrnl. Appl. Phys., 36, L899, 1997.

[2] A. Polyakov et al., Phys. Stat. Sol. (a), 207, 6, 1383, 2010.

[3] C. Bayram et al., Appl. Phys. B, 95, 307, 2009. 


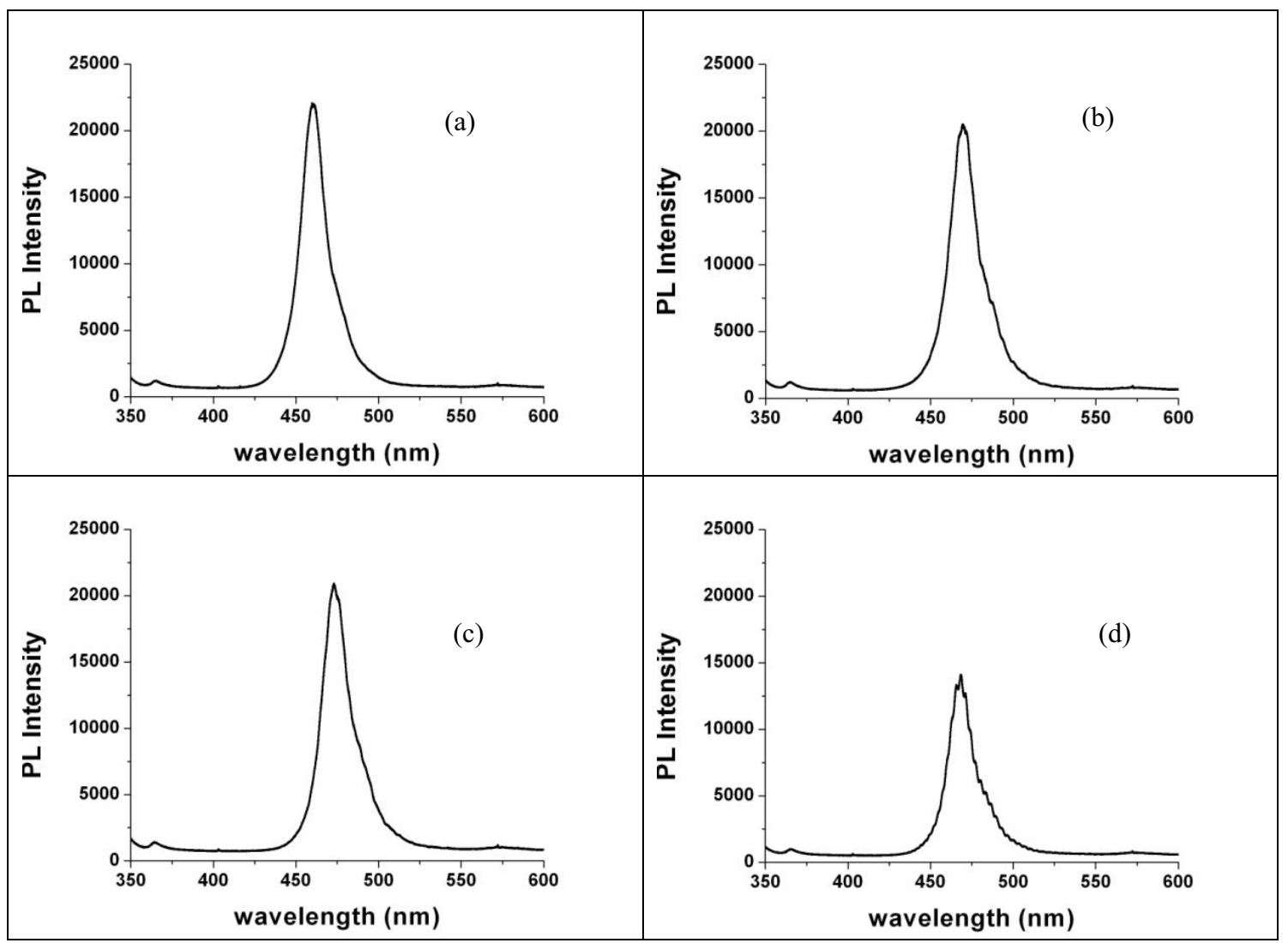

Figure 1. Photoluminescence spectra of our epitaxially-laterally-overgrown InGaN/GaN quantum heterostructures with mask widths of (a) $4 \mu \mathrm{m}$, (b) $7 \mu \mathrm{m}$ and (c) $10 \mu \mathrm{m}$, and (d) reference (negative control group with no pattern).

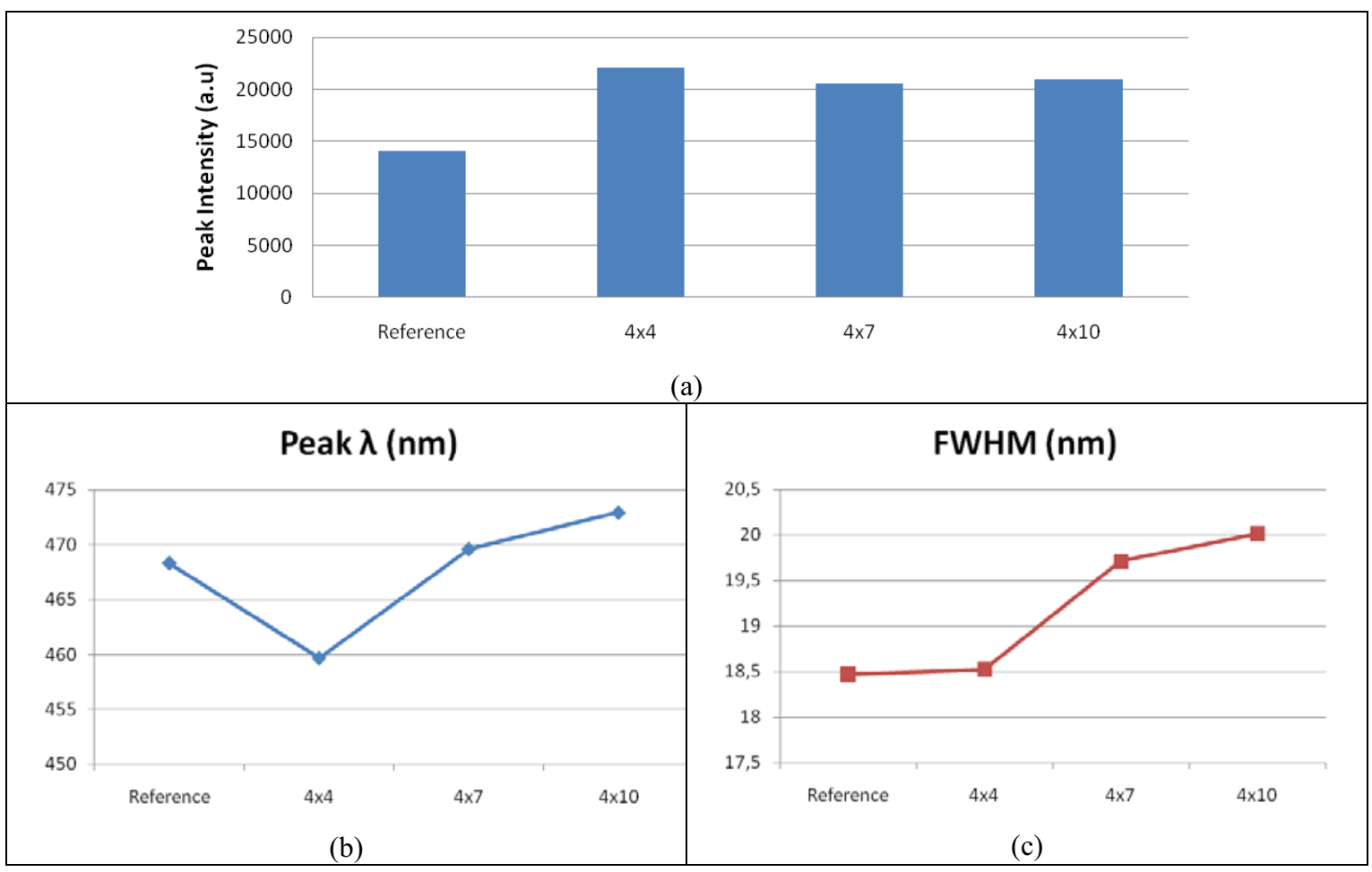

Figure 2. PL spectrum analysis of our ELO heterostructures, (a) peak intensity, (b) peak wavelength, and (c) FWHM. 